\title{
Orlicz norm inequalities for the composite operator and applications
}

Hui $\mathrm{Bi}^{1,2^{*}}$ and Shusen Ding ${ }^{3}$

* Correspondence:

bi_hui2002@yahoo.com.cn

'Department of Applied

Mathematics, Harbin University of

Science and Technology, Harbin,

150080, China

Full list of author information is

available at the end of the article

\begin{abstract}
In this article, we first prove Orlicz norm inequalities for the composition of the homotopy operator and the projection operator acting on solutions of the nonhomogeneous A-harmonic equation. Then we develop these estimates to $L^{\phi}(\mu)$ averaging domains. Finally, we give some specific examples of Young functions and apply them to the norm inequality for the composite operator. 2000 Mathematics Subject Classification: Primary 26B10; Secondary 30C65, 31B10, $46 \mathrm{E} 35$.
\end{abstract}

Keywords: Orlicz norm, the projection operator, the homotopy operator, $L^{\phi ? \varphi}$ ? $(\mu$ ??)averaging domains

\section{Introduction}

Differential forms as the extensions of functions have been rapidly developed. In recent years, some important results have been widely used in PDEs, potential theory, nonlinear elasticity theory, and so forth; see [1-7] for details. However, the study on operator theory of differential forms just began in these several years and hence attracts the attention of many people. Therefore, it is necessary for further research to establish some norm inequalities for operators. The purpose of this article is to establish Orlicz norm inequalities for the composition of the homotopy operator $T$ and the projection operator $H$.

Throughout this article, we always let $E$ be an open subset of $\mathbb{R}^{n}, n \geq 2$. The Lebesgue measure of a set $E \subset \mathbb{R}^{n}$ is denoted by $|E|$. Assume that $B \subset \mathbb{R}^{n}$ is a ball, and $\sigma B$ is the ball with the same center as $B$ and with $\operatorname{diam}(\sigma B)=\sigma \operatorname{diam}(B)$. Let $\Lambda^{k}=\Lambda^{k}\left(\mathbb{R}^{n}\right), k=0,1, \ldots, n$, be the linear space of all $k$-forms $\omega(x)=\sum_{I} \omega_{I}(x) d x_{I}=\sum \omega_{i_{1}, i_{2}, \ldots, i_{k}}(x) d x_{i_{1}} \wedge d x_{i_{2}} \wedge \ldots \wedge d x_{i_{k}}$, where $I=\left(i_{1}, i_{2}, \ldots, i_{k}\right), 1 \leq$ $i_{1}<i_{2}<\ldots<i_{k} \leq n$. We use $\mathcal{D}^{\prime}\left(E, \wedge^{k}\right)$ to denote the space of all differential $k$-forms in $E$. In fact, a differential $k$-form $\omega(x)$ is a Schwarz distribution in $E$ with value in $\wedge^{k}\left(\mathbb{R}^{n}\right)$. As usual, we still use $\star$ to denote the Hodge star operator, and use $d^{\star}: \mathcal{D}^{\prime}\left(E, \wedge^{k+1}\right) \rightarrow \mathcal{D}^{\prime}\left(E, \wedge^{k}\right)$ to denote the Hodge codifferential operator defined by $d^{\star}=(-1)^{n k+1} \star \star \quad d \star \quad$ on $\quad \mathcal{D}^{\prime}\left(E, \wedge^{k+1}\right), k=0,1, \ldots, n-1$. Here $d: \mathcal{D}^{\prime}\left(E, \wedge^{k}\right) \rightarrow \mathcal{D}^{\prime}\left(E, \wedge^{k+1}\right)$ denotes the differential operator.

A weight $w(x)$ is a nonnegative locally integrable function on $\mathbb{R}^{n} . L^{p}\left(E, \Lambda^{k}\right)$ is a Banach space equipped with norm $\|\omega\|_{p, E}=\left(\int_{E}|\omega(x)|^{p} d x\right)^{1 / p}=\left(\int_{E}\left(\sum_{I}\left|\omega_{I}(x)\right|^{2}\right)^{p / 2} d x\right)^{1 / p}$. Let $D$

(c) $2011 \mathrm{Bi}$ and Ding; licensee Springer. This is an Open Access article distributed under the terms of the Creative Commons Attribution License (http://creativecommons.org/licenses/by/2.0), which permits unrestricted use, distribution, and reproduction in any medium, provided the original work is properly cited. 
be a bounded convex domain in $\mathbb{R}^{n}, n \geq 2$, and $C^{\infty}\left(\Lambda^{k} D\right)$ be the space of smooth $k$-forms on $D$, where $\Lambda^{k} D$ is the $k$ th exterior power of the cotangent bundle. The harmonic $k$-field is defined by $\mathcal{H}\left(\wedge^{k} D\right)=\left\{u \in \mathcal{W}\left(\wedge^{k} D\right): d \omega=d^{\star} \omega=0, \omega \in L^{p}\right.$ for some $\left.1<p<\infty\right\}$, where $\mathcal{W}\left(\wedge^{k} D\right)=\left\{\omega \in L_{\text {loc }}^{1}\left(\wedge^{k} D\right): \omega\right.$ has generalized gradient $\}$. If we use $\mathcal{H}^{\perp}$ to denote the orthogonal complement of $\mathcal{H}$ in $L^{1}$, then the Green's operator $G$ is defined by $G: C^{\infty}\left(\wedge^{k} D\right) \rightarrow \mathcal{H}^{\perp} \cap C^{\infty}\left(\wedge^{k} D\right)$ by assigning $G(\omega)$ as the unique element of $\mathcal{H}^{\perp} \cap C^{\infty}\left(\wedge^{k} D\right)$ satisfying $\Delta G(\omega)=\omega-H(\omega)$, where $H$ is the projection operator that maps $C^{\infty}\left(\Lambda^{k} D\right)$ onto $\mathcal{H}$ such that $H(\omega)$ is the harmonic part of $\omega$; see [8] for more properties on the projection operator and Green's operator. The definition of the homotopy operator for differential forms was first introduced in [9]. Assume that $D \subset \mathbb{R}^{n}$ is a bounded convex domain. To each $y \in D$, there corresponds a linear operator $K_{y}: C^{\infty}\left(\wedge^{k} D\right) \rightarrow C^{\infty}\left(\wedge^{k-1} D\right)$ satisfying that $\left(K_{y} \omega\right)\left(x ; \xi_{1}, \xi_{2}, \ldots, \xi_{k-1}\right)=\int_{0}^{1} t^{k-1} \omega\left(t x+y-t y ; x-y, \xi_{1}, \xi_{2}, \ldots, \xi_{k-1}\right) d t$. Then by averaging $K_{y}$ over all points $y$ in $D$, The homotopy operator $T: C^{\infty}\left(\Lambda^{k} D\right) \rightarrow$ $C^{\infty}\left(\wedge^{k-1} D\right)$ is defined by $T \omega=\int_{D} \varphi(\gamma) K_{\gamma} \omega d y$, where $\varphi \in C_{0}^{\infty}(D)$ is normalized so that $\int \phi(y) d y=1$. In [9], those authors proved that there exists an operator $T: L_{l o c}^{1}\left(D, \wedge^{k}\right) \rightarrow L_{l o c}^{1}\left(D, \wedge^{k-1}\right), k=1,2, \ldots, n$, such that

$$
\begin{aligned}
& T(d \omega)+d T \omega=\omega \\
& |T \omega(x)| \leq C \int_{D} \frac{|\omega(y)|}{|y-x|^{n-1}} d y
\end{aligned}
$$

for all differential forms $\omega \in L^{p}\left(D, \Lambda^{k}\right)$ such that $d \omega \in L^{p}\left(D, \wedge^{k}\right)$. Furthermore, we can define the $k$-form $\omega_{D} \in \mathcal{D}^{\prime}\left(D, \wedge^{k}\right)$ by the homotopy operator as

$$
\omega_{D}=|D|^{-1} \int_{D} \omega(y) d y, k=0 ; \omega_{D}=d(T \omega), k=1,2, \ldots, n
$$

for all $\omega \in L^{p}\left(D, \Lambda^{k}\right), 1 \leq p<\infty$.

Consider the nonhomogeneous A-harmonic equation for differential forms

$$
d^{\star} A(x, d \omega)=B(x, d \omega)
$$

where $A: E \times \Lambda^{k}\left(\mathbb{R}^{n}\right) \rightarrow \Lambda^{k}\left(\mathbb{R}^{n}\right)$ and $B: E \times \Lambda^{k}\left(\mathbb{R}^{n}\right) \rightarrow \Lambda^{k-1}\left(\mathbb{R}^{n}\right)$ are two operators satisfying the conditions:

$$
\begin{aligned}
& |A(x, \xi)| \leq a|\xi|^{p-1}, \\
& A(x, \xi) \cdot \xi \geq|\xi|^{p}, \\
& |B(x, \xi)| \leq b|\xi|^{p-1}
\end{aligned}
$$

for almost every $x \in E$ and all $\xi \in \Lambda^{k}\left(\mathbb{R}^{n}\right)$. Here, $a, b>0$ are some constants and $1<$ $p<\infty$ is a fixed exponent associated with (1.4). A solution to (1.4) is an element of the Sobolev space $W_{l o c}^{1, p}\left(E, \wedge^{k-1}\right)$ such that

$$
\int_{E} A(x, d \omega) \cdot d \varphi+B(x, d \omega) \cdot \varphi=0
$$

for all $\varphi \in W_{l o c}^{1, p}\left(E, \wedge^{k-1}\right)$ with compact support. 


\section{Orlicz norm inequalities for the composite operator}

In this section, we establish the weighted inequalities for the composite operator $T \circ H$ in terms of Orlicz norms. To state our results, we need some definitions and lemmas.

We call a continuously increasing function $\Phi:[0, \infty) \rightarrow[0, \infty)$ with $\Phi(0)=0$ an Orlicz function. If the Orlicz function $\Phi$ is convex, then $\Phi$ is often called a Young function. The Orlicz space $L^{\Phi}(E)$ consists of all measurable functions $f$ on $E$ such that $\int_{E} \Phi(|f| / \lambda) d x<\infty$ for some $\lambda=\lambda(f)>0$ with the nonlinear Luxemburg functional

$$
\|f\|_{\Phi, E}=\inf \left\{\lambda>0: \int_{E} \Phi\left(\frac{|f|}{\lambda}\right) d x \leq 1\right\} .
$$

Moreover, if $\Phi$ is a restrictively increasing Young function, then $L^{\Phi}(E)$ is a Banach space and the corresponding norm $\|\cdot\|_{\Phi, E}$ is called Luxemburg norm or Orlicz Norm. The following definition appears in [10].

Definition 2.1. We say that an Orlicz function $\Phi$ lies in the class $G(p, q, C), 1 \leq p<q<\infty$ and $C \geq 1$, if (1) $1 / C \leq \Phi\left(t^{1 / p}\right) / g(t) \leq C$ and (2) $1 / C \leq \Phi\left(t^{1 / q}\right) / h(t) \leq C$ for all $t>0$, where $g(t)$ is a convex increasing function and $h(t)$ is a concave increasing function on $[0, \infty)$.

We note from [10] that each of $\Phi, g$, and $h$ mentioned in Definition 2.1 is doubling, from which it is easy to know that

$$
C_{1} t^{q} \leq h^{-1}(\Phi(t)) \leq C_{2} t^{q}, C_{1} t^{p} \leq g^{-1}(\Phi(t)) \leq C_{2} t^{p}
$$

for all $t>0$, where $C_{1}$ and $C_{2}$ are constants.

We also need the following lemma which appears in [1].

Lemma 2.2. Let $u \in L_{l o c}^{s}\left(D, \wedge^{k}\right), k=1,2, \ldots, n, 1<s<\infty$, be a smooth solution of the nonhomogeneous $A$-harmonic equation in a bounded convex domain $D, H$ be the projection operator and $T: C^{\infty}\left(\Lambda^{k} D\right) \rightarrow C^{\infty}\left(\Lambda^{k-1} D\right)$ be the homotopy operator. Then there exists a constant $C$, independent of $u$, such that

$$
\left\|T(H(u))-(T(H(u)))_{B}\right\|_{s, B} \leq \operatorname{Cdiam}(B)\|u\|_{s, \rho B}
$$

for all balls $B$ with $\rho B \subset D$, where $\rho>1$ is a constant.

The $A_{r}$ weights, $r>1$, were first introduced by Muckenhoupt [11] and play a crucial role in weighted norm inequalities for many operators. As an extension of $A_{r}$ weights, the following class was introduced in [2].

Definition 2.3. We call that a measurable function $w(x)$ defined on a subset $E \subset \mathbb{R}^{n}$ satisfies the $A(\alpha, \beta, \gamma, E)$-condition for some positive constants $\alpha, \beta, \gamma$, write $w(x) \in A(\alpha$, $\beta, \gamma, E)$, if $w(x)>0$ a.e. and

$$
\sup _{B}\left(\frac{1}{|B|} \int_{B} w^{\alpha} d x\right)\left(\frac{1}{|B|} \int_{B}\left(\frac{1}{w}\right)^{\beta} d x\right)^{\gamma / \beta}=c_{\alpha, \beta, \gamma}<\infty,
$$

where the supremum is over all balls $B \subset E$.

We also need the following reverse Hölder inequality for the solutions of the nonhomogeneous A-harmonic equation, which appears in [3].

Lemma 2.4. Let $u$ be a solution of the nonhomogeneous A-harmonic equation, $\sigma>1$ and $0<s, t<\infty$. Then there exists a constant $C$, independent of $u$ and $B$, such that

$$
\left\|\left.u\right|_{s, B} \leq C|B|^{(t-s) / s t}|| u\right\|_{t, \sigma B}
$$


Bi and Ding Journal of Inequalities and Applications 2011, 2011:69

Page 4 of 12

for all balls $B$ with $\sigma B \subset E$.

Theorem 2.5. Assume that $u$ is a smooth solution of the nonhomogeneous A-harmonic equation in a bounded convex domain $D, 1<p, q<\infty$ and $w(x) \in A\left(\alpha, \beta, \frac{\alpha q}{p}\right.$;D)for some $\alpha>1$ and $\beta>0$. Let $H$ be the projection operator and $T: C^{\infty}\left(\wedge^{k} D\right) \rightarrow C^{\infty}\left(\wedge^{k-1} D\right), k=1$, $2, \ldots, n$, be the homotopy operator. Then there exists a constant $C$, independent of $u$, such that

$$
\left(\int_{B}\left|T(H(u))-(T(H(u)))_{B}\right|^{q} w(x) d x\right)^{1 / q} \leq \operatorname{Cdiam}(B)|B|^{(p-q) / p q}\left(\int_{\sigma B}|u|^{p} w(x) d x\right)^{1 / p}
$$

for all balls with $\sigma B \subset D$ for some $\sigma>1$.

Proof. Set $s=\alpha q$ and $m=\beta p /(\beta+1)$. From Lemma 2.2 and the reverse Hölder inequality, we have

$$
\begin{aligned}
& \left(\int_{B}\left|T(H(u))-(T(H(u)))_{B}\right|^{q} w(x) d x\right)^{1 / q} \\
& \leq\left(\int_{B}\left|T(H(u))-(T(H(u)))_{B}\right|^{\frac{q s}{s-q}} d x\right)^{\frac{s-q}{s q}}\left(\int_{B}(w(x))^{\alpha} d x\right)^{\frac{1}{\alpha q}} \\
& \leq C_{1} \operatorname{diam}(B)|B|^{\frac{1}{q}-\frac{1}{s}-\frac{1}{m}}\left(\int_{\sigma B}|u|^{m} d x\right)^{1 / m}\left(\int_{B}(w(x))^{\alpha} d x\right)^{1 / \alpha q} .
\end{aligned}
$$

Let $n=\frac{p m}{p-m}$, then $\frac{1}{p}+\frac{1}{n}=\frac{1}{m}$. Thus, using the Hölder inequality, we obtain

$$
\begin{aligned}
& \left(\int_{\sigma B}|u|^{m} d x\right)^{1 / m} \\
& =\left(\int_{\sigma B}|u|^{m}\left(w^{\frac{1}{p}} \cdot w^{\frac{-1}{p}}\right) d x\right)^{1 / m} \\
& \leq\left(\int_{\sigma B}|u|^{p} w(x) d x\right)^{1 / p}\left(\int_{\sigma B} w^{\frac{-n}{p}} d x\right)^{\frac{1}{n}} .
\end{aligned}
$$

Note that $w(x) \in A\left(\alpha, \beta, \frac{\alpha q}{p} ; D\right)$. It is easy to find that

$$
\begin{aligned}
& \left(\int_{B}(w(x))^{\alpha} d x\right)^{1 / \alpha q}\left(\int_{\sigma B} w^{\frac{-n}{p}} d x\right)^{\frac{1}{n}} \\
& =\left(\int_{B}(w(x))^{\alpha} d x\right)^{1 / \alpha q}\left(\int_{\sigma B} w^{-\beta} d x\right)^{\frac{1}{\beta p}} \\
& \leq|\sigma B|^{\frac{1}{\mathrm{~s}}+\frac{1}{n}}\left[\left(\frac{1}{|\sigma B|} \int_{\sigma B}(w(x))^{\alpha} d x\right)\left(\frac{1}{|\sigma B|} \int_{\sigma B} w^{-\beta} d x\right)^{\frac{\alpha q}{\beta p}}\right]^{1 / \alpha q} \\
& \leq C_{\alpha, \beta, \frac{\alpha q}{p}}^{1 / \alpha q}|\sigma B|^{\frac{1}{\mathrm{~s}}+\frac{1}{n}} .
\end{aligned}
$$


Combining (2.3)-(2.5) immediately yields that

$$
\begin{aligned}
& \left(\int_{B}\left|T(H(u))-(T(H(u)))_{B}\right|^{q} w(x) d x\right)^{1 / q} \\
& \leq C_{2} \operatorname{diam}(B)|B|^{\frac{1}{q}-\frac{1}{s}-\frac{1}{m}}|\sigma B|^{\frac{1}{s}+\frac{1}{n}}\left(\int_{\sigma B}|u|^{p} w(x) d x\right)^{1 / p} \\
& \leq C_{3} \operatorname{diam}(B)|B|^{(p-q) / p q}\left(\int_{\sigma B}|u|^{p} w(x) d x\right)^{1 / p} .
\end{aligned}
$$

This ends the proof of Theorem 2.5.

If we choose $p=q$ in Theorem 2.5, we have the following corollary.

Corollary 2.6. Assume that $u$ is a solution of the nonhomogeneous A-harmonic equation in a bounded convex domain $D, 1<q<\infty$ and $w(x) \in A(\alpha, \beta, \alpha ; D)$ for some $\alpha>$ 1 and $\beta>0$. Let $H$ be the projection operator and $T: C^{\infty}\left(\wedge^{k} D\right) \rightarrow C^{\infty}\left(\wedge^{k-1} D\right), k=1$, $2, \ldots, n$, be the homotopy operator. Then there exists a constant $C$, independent of $u$, such that

$$
\left(\int_{B}\left|T(H(u))-(T(H(u)))_{B}\right|^{q} w(x) d x\right)^{1 / q} \leq \operatorname{Cdiam}(B)\left(\int_{\sigma B}|u|^{q} w(x) d x\right)^{1 / q}
$$

for all balls with $\sigma B \subset D$ for some $\sigma>1$.

Next, we prove the following inequality, which is a generalized version of the one given in Lemma 2.2. More precisely, the inequality in Lemma 2.2 is a special case of the following result when $\phi(t)=t^{p}$.

Theorem 2.7. Assume that $\phi$ is a Young function in the class $G\left(p, q, C_{0}\right), 1<p<q$ $<\infty, C_{0} \geq 1$ and $D$ is a bounded convex domain. If $u \in C^{\infty}\left(\wedge^{k} D\right), k=1,2, \ldots, n$, is a solution of the nonhomogeneous A-harmonic equation in $D, \varphi(|u|) \in L_{l o c}^{1}(D, d x)$ and $1 / p-1 /$ $q \leq 1 / n$, then there exists a constant $C$, independent of $u$, such that

$$
\int_{B} \varphi\left(\left|T(H(u))-(T(H(u)))_{B}\right|\right) d x \leq C \int_{\sigma B} \varphi(|u|) d x
$$

for all balls $B$ with $\sigma B \subset D$, where $\sigma>1$ is a constant.

Proof. From Lemma 2.2, we know that

$$
\left\|T(H(u))-(T(H(u)))_{B}\right\|_{s, B} \leq C_{1} \operatorname{diam}(B)\|u\|_{s, \sigma B}
$$

for $1<s<\infty$. Note that $u$ is a solution of the nonhomogeneous A-harmonic equation. Hence, by the reverse Hölder inequality, we have

$$
\begin{aligned}
& \left(\int_{B}\left|T(H(u))-(T(H(u)))_{B}\right|^{q} d x\right)^{1 / q} \\
& \leq C_{1} \operatorname{diam}(B)\left(\int_{\sigma_{1} B}|u|^{q} d x\right)^{1 / q} \\
& \leq C_{2} \operatorname{diam}(B)\left|\sigma_{1} B\right|^{(p-q) / p q}\left(\int_{\sigma_{2} B}|u|^{p} d x\right)^{1 / p},
\end{aligned}
$$

where $\sigma_{2}>\sigma_{1}>1$ are some constants. Thus, using that $\phi$ and $g$ are increasing functions as well as Jensen's inequality for $g$, we deduce that 


$$
\begin{aligned}
& \varphi\left(\left(\int_{B}\left|T(H(u))-(T(H(u)))_{B}\right|^{q} d x\right)^{1 / q}\right) \\
& \leq \varphi\left(C_{2} \operatorname{diam}(B)\left|\sigma_{1} B\right|^{(p-q) / p q}\left(\int_{\sigma_{2} B}|u|^{p} d x\right)^{1 / p}\right) \\
& \leq \varphi\left(\left(C_{2}^{p}(\operatorname{diam}(B))^{p}\left|\sigma_{1} B\right|^{(p-q) / q} \int_{\sigma_{2} B}|u|^{p} d x\right)^{1 / p}\right) \\
& \leq C_{3} g\left(C_{2}^{p}(\operatorname{diam}(B))^{p}\left|\sigma_{1} B\right|^{(p-q) / q} \int_{\sigma_{2} B}|u|^{p} d x\right) \\
& =C_{3} g\left(\int_{\sigma_{2} B} C_{2}^{p}(\operatorname{diam}(B))^{p}\left|\sigma_{1} B\right|^{(p-q) / q}|u|^{p} d x\right) \\
& \leq C_{3} \int_{\sigma_{2} B} g\left(C_{2}^{p}(\operatorname{diam}(B))^{p}\left|\sigma_{1} B\right|^{(p-q) / q}|u|^{p}\right) d x .
\end{aligned}
$$

Since $1 / p-1 / q \leq 1 / n$, we have

$$
\operatorname{diam}(B)\left|\sigma_{1} B\right|^{\frac{p-q}{p q}} \leq C_{4}|D|^{\frac{1}{n}+\frac{1}{q}-\frac{1}{p}} \leq C_{5} .
$$

Applying (2.7) and (2.8) and noting that $g(t) \leq C_{0} \phi\left(t^{1 / p}\right)$, we have

$$
\begin{aligned}
& \int_{\sigma_{2} B} g\left(C_{2}^{p}(\operatorname{diam}(B))^{p}\left|\sigma_{1} B\right|^{(p-q) / q}|u|^{p}\right) d x \\
& \leq C_{0} \int_{\sigma_{2} B} \varphi\left(C_{2} \operatorname{diam}(B)\left|\sigma_{1} B\right|^{(p-q) / p q}|u|\right) d x \\
& \leq C_{0} \int_{\sigma_{2} B} \varphi\left(C_{6}|u|\right) d x .
\end{aligned}
$$

It follows from (2.7) and (2.9) that

$$
\begin{aligned}
& \varphi\left(\left(\int_{B}\left|T(H(u))-(T(H(u)))_{B}\right|^{q} d x\right)^{1 / q}\right) \\
& \leq C_{7} \int_{\sigma_{2} B} \varphi\left(C_{6}|u|\right) d x .
\end{aligned}
$$

Applying Jensen's inequality once again to $h^{-1}$ and considering that $\phi$ and $h$ are doubling, we have

$$
\begin{aligned}
& \int_{B} \varphi\left(\left|T(H(u))-(T(H(u)))_{B}\right|\right) d x \\
& =h\left(h^{-1}\left(\int_{B} \varphi\left(\left|T(H(u))-(T(H(u)))_{B}\right|\right) d x\right)\right) \\
& \leq h\left(\int_{B} h^{-1}\left(\varphi\left(\left|T(H(u))-(T(H(u)))_{B}\right|\right) d x\right)\right) \\
& \leq h\left(C_{8} \int_{B}\left|T(H(u))-(T(H(u)))_{B}\right|^{q} d x\right) \\
& \leq C_{0} \varphi\left(\left(C_{8} \int_{B}\left|T(H(u))-(T(H(u)))_{B}\right|^{q} d x\right)^{1 / q}\right) \\
& \leq C_{9} \int_{\sigma_{2} B} \varphi\left(C_{6}|u|\right) d x \\
& \leq C_{10} \int_{\sigma_{2} B} \varphi(|u|) d x .
\end{aligned}
$$

This ends the proof of Theorem 2.7. 
To establish the weighted version of the inequality obtained in the above Theorem 2.7, we need the following lemma which appears in [4].

Lemma 2.8. Let $u$ be a solution of the nonhomogeneous A-harmonic equation in a domain $E$ and $0<p, q<\infty$. Then, there exists a constant $C$, independent of $u$, such that

$$
\left(\int_{B}|u|^{q} d \mu\right)^{1 / q} \leq C(\mu(B))^{\frac{p-q}{p q}}\left(\int_{\sigma B}|u|^{p} d \mu\right)^{1 / p}
$$

for all balls $B$ with $\sigma B \subset E$ for some $\sigma>1$, where the Radon measure $\mu$ is defined by $d \mu=w(x) d x$ and $w \in A(\alpha, \beta, \alpha ; E), \alpha>1, \beta>0$.

Theorem 2.9. Assume that $\phi$ is a Young function in the class $G\left(p, q, C_{0}\right), 1<p<q$ $<\infty, C_{0} \geq 1$ and $D$ is a bounded convex domain. Let $d \mu=w(x) d x$, where $w(x) \in A(\alpha, \beta$, $\alpha$; D) for $\alpha>1$ and $\beta>0$. If $u \in C^{\infty}\left(\wedge^{k} D\right), k=1,2, \ldots, n$, is a solution of the nonhomogeneous A-harmonic equation in $D, \varphi(|u|) \in L_{l o c}^{1}(D, d \mu)$, then there exists a constant $C$, independent of $u$, such that

$$
\int_{B} \varphi\left(\left|T(H(u))-(T(H(u)))_{B}\right|\right) d \mu \leq C \int_{\sigma B} \varphi(|u|) d \mu
$$

for all balls $B$ with $\sigma B \subset D$ and $|B| \geq d_{0}>0$, where $\sigma>1$ is a constant.

Proof. From Corollary 2.6 and Lemma 2.8, we have

$$
\begin{aligned}
& \left(\int_{B}\left|T(H(u))-(T(H(u)))_{B}\right|^{q} d \mu\right)^{1 / q} \\
& \leq C_{1} \operatorname{diam}(B)\left(\int_{\sigma_{1} B}|u|^{q} d \mu\right)^{1 / q} \\
& \leq C_{2} \operatorname{diam}(B)(\mu(B))^{(p-q) / p q}\left(\int_{\sigma_{2} B}|u|^{p} d \mu\right)^{1 / p},
\end{aligned}
$$

where $\sigma_{2}>\sigma_{1}>1$ is some constant. Note that $\phi$ and $g$ are increasing functions and $g$ is convex in $D$. Hence by Jensen's inequality for $g$, we deduce that

$$
\begin{aligned}
& \varphi\left(\left(\int_{B}\left|T(H(u))-(T(H(u)))_{B}\right|^{q} d \mu\right)^{1 / q}\right) \\
& \leq \varphi\left(C_{2} \operatorname{diam}(B)(\mu(B))^{(p-q) / p q}\left(\int_{\sigma_{2} B}|u|^{p} d \mu\right)^{1 / p}\right) \\
& =\varphi\left(\left(C_{2}^{p}(\operatorname{diam}(B))^{p}(\mu(B))^{(p-q) / q} \int_{\sigma_{2} B}|u|^{p} d \mu\right)^{1 / p}\right) \\
& \leq C_{3} g\left(C_{2}^{p}(\operatorname{diam}(B))^{p}(\mu(B))^{(p-q) / q} \int_{\sigma_{2} B}|u|^{p} d \mu\right) \\
& =C_{3} g\left(\int_{\sigma_{2} B} C_{2}^{p}(\operatorname{diam}(B))^{p}(\mu(B))^{(p-q) / q}|u|^{p} d \mu\right) \\
& \leq C_{3} \int_{\sigma_{2} B} g\left(C_{2}^{p}(\operatorname{diam}(B))^{p}(\mu(B))^{(p-q) / q}|u|^{p}\right) d \mu .
\end{aligned}
$$

Set $D_{1}=\{x \in D: 0<w(x)<1\}$ and $D_{2}=\{x \in D: w(x) \geq 1\}$. Then $D=D_{1} \cup D_{2}$. We let $\tilde{w}(x)=1$, if $x \in D_{1}$ and $\tilde{w}(x)=w(x)$, if $x \in D_{2}$. It is easy to check that $w(x) \in A(\alpha$, $\beta, \alpha ; D)$ if and only if $\tilde{w}(x) \in A(\alpha, \beta, \alpha ; D)$. Thus, we may always assume that $w(x) \geq 1$ a.e. in $D$. Hence, we have $\mu(B)=\int_{B} w(x) d x \geq|B|$ for all balls $B \subset D$. Since $p<q$ and $\mid$ 
$B \mid=d_{0}>0$, it is easy to find that

$$
\operatorname{diam}(B) \mu(B)^{(p-q) / p q} \leq \operatorname{diam}(D) d_{0}^{(p-q) / p q} \leq C_{3}
$$

It follows from (2.13) and $g(t) \leq C_{0} \phi\left(t^{1 / p}\right)$ that

$$
\begin{aligned}
& \int_{\sigma_{2} B} g\left(C_{2}^{p}(\operatorname{diam}(B))^{p}(\mu(B))^{(p-q) / q}|u|^{p}\right) d \mu \\
& \leq C_{0} \int_{\sigma_{2} B} \varphi\left(C_{2} \operatorname{diam}(B)(\mu(B))^{(p-q) / p q}|u|\right) d \mu \\
& \leq C_{0} \int_{\sigma_{2} B} \varphi\left(C_{4}|u|\right) d \mu .
\end{aligned}
$$

Applying Jensen's inequality to $h^{-1}$ and considering that $\phi$ and $h$ are doubling, we have

$$
\begin{aligned}
& \int_{B} \varphi\left(\left|T(H(u))-(T(H(u)))_{B}\right|\right) d \mu \\
& =h\left(h^{-1}\left(\int_{B} \varphi\left(\left|T(H(u))-(T(H(u)))_{B}\right|\right) d \mu\right)\right) \\
& \leq h\left(\int_{B} h^{-1}\left(\varphi\left(\left|T(H(u))-(T(H(u)))_{B}\right|\right) d \mu\right)\right) \\
& \leq h\left(C_{8} \int_{B}\left|T(H(u))-(T(H(u)))_{B}\right|^{q} d \mu\right) \\
& \leq C_{0} \varphi\left(\left(C_{8} \int_{B}\left|T(H(u))-(T(H(u)))_{B}\right|^{q} d \mu\right)^{1 / q}\right) \\
& \leq C_{9} \int_{\sigma_{2} B} \varphi\left(C_{6}|u|\right) d \mu \\
& \leq C_{10} \int_{\sigma_{2} B} \varphi(|u|) d \mu .
\end{aligned}
$$

This ends the proof of Theorem 2.9.

Note that if we remove the restriction on balls $B$, then we can obtain a weighted inequality in the class $A\left(\alpha, \beta, \frac{\alpha q}{p} ; D\right)$, for which the method of proof is analogous to the one in Theorem 2.9. We now give the statement as follows.

Theorem 2.10. Assume that $\phi$ is a Young function in the class $G\left(p, q, C_{0}\right), 1<p<q$ $<\infty, C_{0} \geq 1$ and $D$ is a bounded convex domain. Let $d \mu=w(x) d x$, where $w(x) \in A\left(\alpha, \beta, \frac{\alpha q}{p} ; D\right)$ for $\alpha>1$ and $\beta>0$. If $u \in C^{\infty}\left(\wedge^{k} D\right), k=1,2, \ldots, n$, is a solution of the nonhomogeneous A-harmonic equation in $D, \varphi(|u|) \in L_{l o c}^{1}(D, d \mu)$ and $1 / p-1 / q \leq 1 /$ $n$, then there exists a constant $C$, independent of $u$, such that

$$
\int_{B} \varphi\left(\left|T(H(u))-(T(H(u)))_{B}\right|\right) d \mu \leq C \int_{\sigma B} \varphi(|u|) d \mu
$$

for all balls $B$ with $\sigma B \subset D$, where $\sigma>1$ is a constant.

Directly from the proof of Theorem 2.7, if we replace $\left|T(H(u))-(T(H(u)))_{B}\right|$ by $\frac{1}{\lambda}\left|T(H(u))-(T(H(u)))_{B}\right|$, then we immediately have

$$
\int_{B} \varphi\left(\frac{\left|T(H(u))-(T(H(u)))_{B}\right|}{\lambda}\right) d x \leq C \int_{\sigma B} \varphi\left(\frac{|u|}{\lambda}\right) d x
$$


for all balls $B$ with $\sigma B \subset D$ and $\lambda>0$. Furthermore, from the definition of the Orlicz norm and (2.15), the following Orlicz norm inequality holds.

Corollary 2.11. Assume that $\phi$ is a Young function in the class $G\left(p, q, C_{0}\right), 1<p<q$ $<\infty, C_{0} \geq 1$ and $D$ is a bounded convex domain. If $u \in C^{\infty}\left(\wedge^{k} D\right), k=1,2, \ldots, n$, is a solution of the nonhomogeneous A-harmonic equation in $D, \varphi(|u|) \in L_{l o c}^{1}(D, d x)$ and $1 / p-1 /$ $q \leq 1 / n$, then there exists a constant $C$, independent of $u$, such that

$$
\left\|T(H(u))-(T(H(u)))_{B}\right\|_{\varphi, B} \leq C\|u\|_{\varphi, \sigma B}
$$

for all balls $B$ with $\sigma B \subset D$, where $\sigma>1$ is a constant.

Next, we extend the local Orlicz norm inequality for the composite operator to the global version in the $L^{\phi}(\mu)$-averaging domains.

In [12], Staples introduced $L^{s}$-averaging domains in terms of Lebesgue measure. Then, Ding and Nolder [6] developed $L^{s}$-averaging domains to weighted versions and obtained a similar characterization. At the same time, they also established a global norm inequality for conjugate A-harmonic tensors in $L^{s}(\mu)$-averaging domains. In the following year, Ding [5] further generalized $L^{s}$-averaging domains to $L^{\phi}(\mu)$-averaging domains, for which $L^{s}(\mu)$-averaging domains are special cases when $\phi(t)=t^{s}$. The following definition appears.

Definition 2.12. Let $\phi$ be an increasing convex function defined on $[0, \infty)$ with $\phi(0)=$ 0 . We say a proper subdomain $\Omega \subset \mathbb{R}^{n}$ an $L^{\phi}(\mu)$-averaging domain, if $\mu(\Omega)<\infty$ and there exists a constant $C$ such that

$$
\int_{\Omega} \varphi\left(\tau\left|u-u_{B_{0}}\right|\right) d \mu \leq \underset{B}{C \sup _{B}} \int_{B} \varphi\left(\sigma\left|u-u_{B}\right|\right) d \mu
$$

for some balls $B_{0} \subset \Omega$ and all $u$ such that $\varphi(|u|) \in L_{\text {loc }}^{1}(\Omega, d \mu)$, where $0<\tau, \sigma<\infty$ are constants and the supremum is over all balls $B \subset \Omega$.

Theorem 2.13. Let $\phi$ be a Young function in the class $G\left(p, q, C_{0}\right), 1<p<q<\infty, C_{0}$ $\geq 1$ and $D$ is a bounded convex $L^{\phi}(d x)$-averaging domain. Suppose that $\phi(|u|) \in L^{1}(D$, $d x), u \in C^{\infty}\left(\wedge^{1} D\right)$ is a solution of the nonhomogeneous $A$-harmonic equation in $D$ and $1 / p-1 / q \leq 1 / n$. Then there exists a constant $C$, independent of $u$, such that

$$
\int_{D} \varphi\left(\left|T(H(u))-(T(H(u)))_{B_{0}}\right|\right) d x \leq C \int_{D} \varphi(|u|) d x,
$$

where $B_{0} \subset D$ is a fixed ball.

Proof. Since $D$ is an $L^{\phi}(d x)$-averaging domain and $\phi$ is doubling, from Theorem 2.7, we have

$$
\begin{aligned}
& \int_{D} \varphi\left(\left|T(H(u))-(T(H(u)))_{B_{0}}\right|\right) d x \\
& \leq C_{1} \sup _{B \subset D} \int_{B} \varphi\left(\left|T(H(u))-(T(H(u)))_{B}\right|\right) d x \\
& \leq C_{1} \sup _{B \subset D}\left(C_{2} \int_{\sigma B} \varphi(|u|) d x\right) \\
& \leq C_{3} \int_{D} \varphi(|u|) d x .
\end{aligned}
$$

We have completed the proof of Theorem 2.13. 
Clearly, (2.17) implies that

$$
\left\|T(H(u))-(T(H(u)))_{B_{0}}\right\|_{\varphi, D} \leq C\|u\|_{\varphi, D} .
$$

Similarly, we also can develop the inequalities established in Theorems 2.9 and 2.10 to $L^{\phi}(\mu)$-averaging domains, for which $d \mu=w(x) d x$ and $w(x) \in A(\alpha, \beta, \alpha ; D)$ and $A\left(\alpha, \beta, \frac{\alpha q}{p} ; D\right)$, respectively.

\section{Applications}

The homotopy operator provides a decomposition to differential forms $\omega \in L^{p}\left(D, \Lambda^{k}\right)$ such that $d \omega \in L^{p}\left(D, \Lambda^{k+1}\right)$. Sometimes, however, the expression of $T(H(u))$ or $(T H(u))_{B}$ may be quite complicated. However, using the estimates in the previous section, we can obtain the upper bound for the Orlicz norms of $T(H(u))$ or $(T H(u))_{B}$. In this section, we give some specific estimates for the solutions of the nonhomogeneous A-harmonic equation. Meantime, we also give several Young functions that lie in the class $G(p, q, C)$ and then establish some corresponding norm inequalities for the composite operator.

In fact, the nonhomogeneous A-harmonic equation is an extension of many familiar equations. Let $B=0$ and $u$ be a 0 -form in the nonhomogeneous A-harmonic equation (1.4). Thus, (1.4) reduces to the usual A-harmonic equation:

$$
\operatorname{div} A(x, \nabla u)=0 .
$$

In particular, if we take the operator $A(x, \xi)=\xi|\xi|^{p-2}$, then Equation 3.1 further reduces to the $p$-harmonic equation

$$
\operatorname{div}\left(\nabla u|\nabla u|^{p-2}\right)=0 .
$$

It is easy to verify that the famous Laplace equation $\Delta u=0$ is a special case of $p=2$ to the $p$-harmonic equation.

In $\mathbb{R}^{3}$, consider that

$$
\omega=\frac{1}{r^{3}}\left(x_{1} d x_{2} \wedge d x_{3}+x_{2} d x_{3} \wedge d x_{1}+x_{3} d x_{1} \wedge d x_{2}\right),
$$

where $r=\sqrt{x_{1}^{2}+x_{2}^{2}+x_{3}^{2}}$. It is easy to check that $d \omega=0$ and $|\omega|=\frac{1}{r^{2}} \mid$. Hence, $\omega$ is a solution of the nonhomogeneous A-harmonic equation. Let $B$ be a ball with the origin $O \notin \sigma B$, where $\sigma>1$ is a constant. Usually the term $\int_{B} \varphi\left(\left|T(H(\omega))-(T(H(\omega)))_{B}\right|\right) d x$ is not easy to estimate due to the complexity of the operators $T$ and $H$ as well as the function $\phi$. However, by Theorem 2.7, we can give an upper bound of Orlicz norm. Specially, if the Young function $\phi$ is not very complicated, sometimes it is possible to obtain a specific upper bound. For instance, take $\phi(t)=t^{p} \log _{+} t$, where $\log _{+} t=1$ if $t \leq$ $e$ and $\log _{+} t=\log t$ if $t>e$. It is easy to verify that $\phi(t)=t^{p} \log _{+} t$ is a Young function and belongs to $G\left(p_{1}, p_{2}, C\right)$ for some constant $C=C\left(p_{1}, p_{2}, p\right)$. Let $0<M<\infty$ be the upper bound of $|\omega|$ in $\sigma B$. Thus, we have

$$
\begin{aligned}
& \int_{B}\left|T(H(\omega))-(T(H(\omega)))_{B}\right|^{p} \log _{+}\left|T(H(\omega))-(T(H(\omega)))_{B}\right| d x \\
& \leq \int_{\sigma B}|\omega|^{p} \log _{+}(|\omega|) d x \leq \int_{\sigma B} M^{p} \log _{+} M d x=M^{p} \log _{+} M|\sigma B|,
\end{aligned}
$$


where $\sigma>1$ is some constant. Also, if we let $\phi(t)=t^{p} \log _{+} t$ in Theorem 2.13, we can obtain a global estimate in a bounded convex $L^{\phi}(d x)$-averaging domain $\mathrm{D}$ without the origin. That is

$$
\begin{aligned}
& \int_{D}\left|T(H(\omega))-(T(H(\omega)))_{B_{0}}\right|^{p} \log _{+}\left|T(H(\omega))-(T(H(\omega)))_{B_{0}}\right| d x \\
& \leq \int_{D}|\omega|^{p} \log _{+}(|\omega|) d x \leq \int_{D} N^{p} \log _{+} N d x=N^{p} \log _{+} N|D|,
\end{aligned}
$$

where $B_{0} \subset D$ is a fixed ball and $N$ is the upper bound of $|\omega|$ in $D$.

Next we give some examples of Young functions that lie in $G(p, q, C)$ and then apply them to Theorem 2.9 .

Consider the function $\Psi(t)=t^{p} \log _{+}^{\alpha} t, 1<p<\infty, \alpha \in \mathbb{R}$. Obviously, if we take $\alpha=1$, then $\Psi(t)$ reduces to $\phi(t)=t^{p} \log _{+} t$ mentioned above. It is easy to check that for all 1 $\leq p_{1}<p<p_{2}<\infty$ and $\alpha \in \mathbb{R}$, the function $\Psi(t) \in G\left(p_{1}, p_{2}, C\right)$, where $C$ is dependent on $p, p_{1}, p_{2}$ and $\alpha$. However, $\Psi(t)$ is not always a Young function. More precisely, $\Psi$ $(t)$ cannot guarantee to be both increasing and convex. However, note that for $\Psi(t)$, we can always find $K>1$ depending on $p$ and $\alpha$ such that the function $\Psi(t)$ is increasing and convex on both $[0,1]$ and $[K, \infty)$. Furthermore, if let $\Psi_{K}(t)=\Psi(t)$ on $[0,1] \cup$ $[K, \infty)$ and $\Psi_{K}(t)=\Psi(1)+\frac{\Psi(K)-\Psi(1)}{K-1}(t-1)$ in $(1, K)$, then $\Psi_{K}(t)$ still lies in $G\left(p_{1}, p_{2}\right.$, $C)$ for some $C=C\left(p, \alpha, p_{1}, p_{2}\right)$. It is worth noting that after such modification $\Psi_{K}(t)$ is convex in the entire interval $[0, \infty)$, in the sense that $\Psi_{K}(t)$ is a Young function that lies in the class $G(p, q, C)$; see [10] for more details on $\Psi_{K}(t)$. Thus, we have the following result.

Corollary 3.1. Assume that $u \in C^{\infty}\left(\Lambda^{k} D\right), k=1,2, \ldots, n$, is a solution of the nonhomogeneous A-harmonic equation in $D$, where $D$ is a bounded convex domain. Let $d \mu=w$ $(x) d x$ and $\Psi_{K}(|u|) \in L_{l o c}^{1}(D, d \mu)$, where $w(x) \in A(\alpha, \beta, \alpha ; D)$ for $\alpha>1$ and $\beta>0$. Then, for the composition of the homotopy operator $T$ and the projection operator $H$, we have

$$
\int_{B} \Psi_{K}\left(\left|T(H(u))-(T(H(u)))_{B}\right|\right) d \mu \leq C \int_{\sigma B} \Psi_{K}(|u|) d \mu
$$

for all balls $B$ with $\sigma B \subset D$ and $|B| \geq d_{0}>0$. Here $\sigma$ and $C$ are constants and $C$ is independent of $u$.

For the other example consider the function $\Phi(t)=t^{p} \sin t$, on $\left[0, \frac{\pi}{2}\right]$ and $\Phi(t)=t^{p}$, in $\left(\frac{\pi}{2}, \infty\right), 3<p<\infty$. It is easy to check that $\Phi(t)$ is a Young function and for all $0<$ $p_{1}<p+1<p_{2}<\infty, \Phi(t) \in G\left(p_{1}, p_{2}, C\right)$, where $C=C\left(p, p_{1}, p_{2}\right) \geq 1$ is some constant. Thus, Theorem 2.9 holds for $\Phi(t)$ and we have the following corollary.

Corollary 3.2. Assume that $u \in C^{\infty}\left(\wedge^{k} D\right), k=1,2, \ldots, n$, is a solution of the nonhomogeneous A-harmonic equation in $D$, where $D$ is a bounded convex domain. Let $d \mu=w$ $(x) d x$ and $\Phi(|u|) \in L_{l o c}^{1}(D, d \mu)$, where $w(x) \in A(\alpha, \beta, \alpha ; D)$ for $\alpha>1$ and $\beta>0$. Then, for the composition of the homotopy operator $T$ and the projection operator $H$, we have

$$
\int_{B} \Phi\left(\left|T(H(u))-(T(H(u)))_{B}\right|\right) d \mu \leq C \int_{\sigma B} \Phi(|u|) d \mu
$$

for all balls $B$ with $\sigma B \subset D$ and $|B| \geq d_{0}>0$. Here $\sigma$ and $C$ are constants and $C$ is independent of $u$. 


\section{Acknowledgements}

The authors express their sincere thanks to the referee for his/her thoughtful suggestions. H.B. was supported by the Foundation of Education Department of Heilongjiang Province in 2011 (\#12511111) and by the Youth Foundation at the Harbin University of Science and Technology (\# 2009YF033).

\section{Author details}

${ }^{1}$ Department of Applied Mathematics, Harbin University of Science and Technology, Harbin, 150080, China

${ }^{2}$ Department of Mathematics, Harbin Institute of Technology, Harbin, 150001, China ${ }^{3}$ Department of Mathematics,

Seattle University, Seattle, WA 98122, USA

\section{Authors' contributions}

$\mathrm{HB}$ and SD jointly contributed to the main results and HB wrote the paper. All authors read and approved the final manuscript.

\section{Competing interests}

The authors declare that they have no competing interests.

Received: 8 March 2011 Accepted: 24 September 2011 Published: 24 September 2011

\section{References}

1. Agarwal, RP, Ding, S, Nolder, CA: Inequalities for Differential Forms. Springer, New York. (2009)

2. Xing, Y, Ding, S: A new weight class and Poincaré inequalities with the Radon measures. (preprint)

3. Nolder, CA: Global integrability theorems for A-harmonic tensors. J Math Anal Appl. 247, 236-245 (2000). doi:10.1006/ jmaa.2000.6850

4. Xing, Y, Ding, S: Caccioppoli inequalities with Orlicz norms for solutions of harmonic equations and applications. Nonlinearity. 23, 1109-1119 (2010). doi:10.1088/0951-7715/23/5/005

5. Ding, S: $L^{\varphi}(\mu)$-averaging domains and the quasi-hyperbolic metric. Comput Math Appl. 47, 1611-1618 (2004). doi:10.1016/j.camwa.2004.06.016

6. Ding, S, Nolder, CA: $L^{5}(\mu)$-averaging domains. J Math Anal Appl. 283, 85-99 (2003). doi:10.1016/S0022-247X(03)00216-6

7. Ding, S, Liu, B: A singular integral of the composite operator. Appl Math Lett. 22, 1271-1275 (2009). doi:10.1016/j. aml.2009.01.041

8. Warner, FW: Foundations of Differentiable Manifolds and Lie Groups. Springer, New York (1983)

9. Iwaniec, T, Lutoborski, A: Integral estimates for null Lagrangians. Arch Rational Mech Anal. 125, 25-79 (1993). doi:10.1007/BF00411477

10. Buckley, SM, Koskela, P: Orlicz-Hardy inequalities. Illinois J Math. 48, 787-802 (2004)

11. Muckenhoupt, B: weighted norm inequalities for the Hardy-Littlewood maximal operator. Trans Am Math Soc. 165 , 207-226 (1972)

12. Staples, SG: $L^{p}$-averaging domains and the Poincaré inequality. Ann Acad Sci Fenn Ser A I Math. 14, 103-127 (1989)

doi:10.1186/1029-242X-2011-69

Cite this article as: $\mathrm{Bi}$ and Ding: Orlicz norm inequalities for the composite operator and applications. Journal of Inequalities and Applications 2011 2011:69.

\section{Submit your manuscript to a SpringerOpen ${ }^{\circ}$ journal and benefit from:}

- Convenient online submission

- Rigorous peer review

- Immediate publication on acceptance

- Open access: articles freely available online

- High visibility within the field

- Retaining the copyright to your article

Submit your next manuscript at $\boldsymbol{s p r i n g e r o p e n . c o m ~}$ 